\title{
ULTRA-HIGH Q MONOCRYSTALLINE SILICON CARBIDE DISK RESONATORS ANCHORED UPON A PHONONIC CRYSTAL
}

\author{
Jeremy Yang, Benoit Hamelin, Seung-Deok Ko, and Farrokh Ayazi
}

Georgia Institute of Technology, Atlanta, Georgia, USA

\begin{abstract}
This work introduces a 3D design incorporating a phononic crystal to decouple a centrally-supported silicon carbide bulk acoustic wave disk resonator from its $\mathrm{Si}$ handle layer. By overcoming thermoelastic and anchor losses in bulk acoustic wave resonators, this scheme allows for probing the phonon-phonon dissipation limits i.e. Akhiezer loss. Using a custom silicon carbide on insulator platform, $\mathrm{SiC}$ disk resonators anchored upon phononic crystal were fabricated and optically measured. $\mathrm{SiC}$ disk resonators exhibit $Q$-factors near $4 \mathrm{M}$ with a record $f \cdot Q$ product of $1.85 \times 10^{13} \mathrm{~Hz}$ for the radial breathing mode operating at $5 \mathrm{MHz}$, limited by thermoelastic losses. This is the highest $f \cdot Q$ measured in monocrystalline silicon carbide resonators, an important step toward the batch fabrication of inertial-grade SiC BAW gyroscopes.
\end{abstract}

\section{INTRODUCTION}

The pursuit of ultra-high quality factors $(Q)$ in micro and nano resonators is of great interest and importance, benefiting a wide range of applications. Bulk acoustic wave (BAW) disk resonator gyroscopes offer a host of benefits including shock and vibration resilience, high bandwidth and high $Q$ in moderate vacuum environment, thanks to their relatively high operating frequencies (MHz). With sufficiently high $Q$, inertial grade MEMS gyroscopes in small planar form factor are realizable[1], meeting or potentially surpassing the state of the art. The resonator design needs to surmount energy dissipation mechanisms such as thermoelastic dissipation (TED) and anchor loss (ANC) to reach the Akhiezer limit (AKE) of dissipation. As shown in this paper, with a disk resonator centrally-anchored upon a handle layer composed of a phononic crystal structure (Fig.1), one can largely circumvent anchor losses, scaling a significant barrier toward inertial grade $\mathrm{SiC}$ BAW gyroscopes. Batch fabrication of monocrystalline disk resonators is enabled by custom-made silicon carbide on insulator substrates (SiCOI) [2].

\section{DISSIPATION IN SIC BAW RESONATORS}

An ideal resonator is lossless with an infinite $Q$. Naturally, various physical phenomena exist to siphon energy from the device, limiting its ultimate performance defined by fundamental AKE phonon-phonon interactions. When the phonon distribution is perturbed from its equilibrium distribution by an acoustic wave, normal and Umklapp scattering with thermal phonons act to bring the distribution back to equilibrium[2]. Strongly temperature and material dependent, dissipation is minimal as lattice thermal vibrations are small; conversely, more lattice vibrations at high temperature offer more scattering opportunities. With a large stiffness and low average Grüneisen parameter, silicon carbide is an ideal candidate for ultra-high $Q$ sensors as it possesses an f. $Q_{A K E}$ of $6.4 \times 10^{14} \mathrm{~Hz}$, over $30 \times$ higher than silicon's[3]. However, other dissipation mechanisms must be addressed before hitting Akhiezer limits. Due to the BAW disk resonator's relatively high operating frequency, in vacuum, disks typically suffer from TED and anchor losses.

Thermoelastic dissipation occurs in any material that experiences thermal expansion. Three TED operating regimes exist: isothermal, adiabatic, or maximally dissipative (Debye)[4]. In

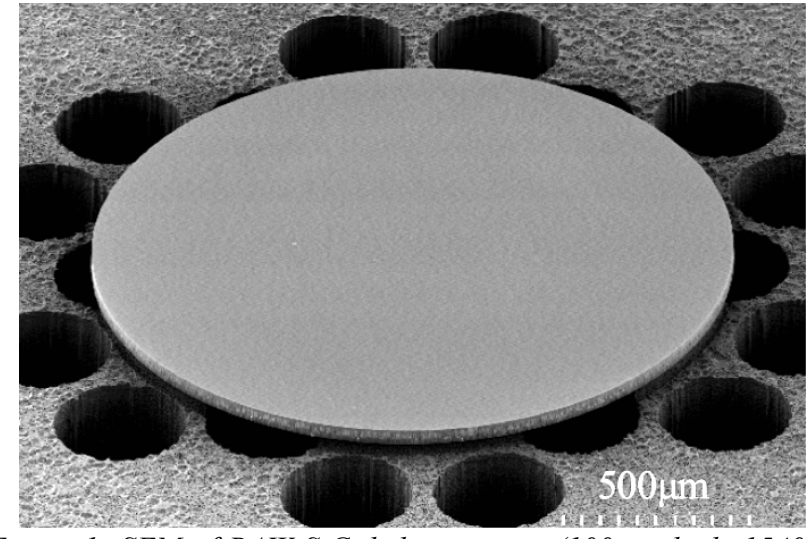

Figure 1: SEM of BAW SiC disk resonator (100 $\mu$ m thick, $1540 \mu \mathrm{m}$ diameter) anchored upon a phononic crystal of three unit cells. The radial mode resonates at $5 \mathrm{MHz}$.

elliptical bulk acoustic wave disk resonators at the MEMS scale, the resonant mechanical frequency is highly disparate from the principal thermal mode frequencies, pushing the device into the deep adiabatic regime, minimizing TED to elevate $Q_{\mathrm{TED}}$ to $14 \mathrm{M}$ for the breathing mode (Fig.2) in FEM simulation.

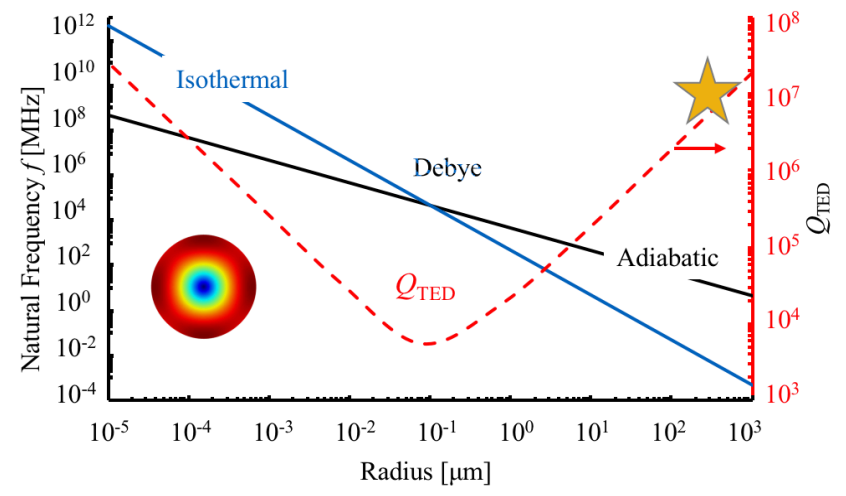

Figure 2: Bulk elliptical modes, the breathing mode in particular (star), of a 1540 $\mu \mathrm{m}$ disk operate adiabatically where $f_{\text {mechanical }}$ (black) $>>f_{\text {thermal }}$ (blue). Minimum $Q_{T E D}($ red) occurs at the Debye frequency. The breathing mode and $m=3$ modes possess $Q_{T E D}$ of $14 M$ and $50 M$, respectively.

Anchor loss remains as one of the most conspicuous sources of loss, adding unpredictability to the limits of quality factor. In centrally-anchored disk resonators, the suppression of anchor loss is particularly difficult due to large elastic strain energy density in the vicinity of the pedestal. While shrinking the size of the pedestal support monotonically mitigates anchor losses, clearly it cannot be made excessively small to ensure resilience against shock and vibration. Similarly, elongation of the pedestal offers a measure of isolation, again compromising structural integrity. Substratedecoupling elements may be incorporated into the device layer[5][6]; however, this comes at the expense of increased TED due to additional strain at the sites of the occlusions. Therefore, it's imperative to implement a $3 \mathrm{D}$ design in which the substrate- 
decoupling elements lie in the handle layer beneath the disk, leaving the disk unencumbered by TED while eliminating anchor losses.

\section{PHONONIC CRYSTAL DECOUPLING}

A phononic crystal $(\mathrm{PnC})$ is a structure composed of stacked acoustically mismatched layers (superlattice) or a tessellation of periodic inclusions in an elastic medium. Analogous to a photonic crystal for electromagnetic radiation, the mismatch of acoustic impedance between the layers or inclusions reflects waves, introducing wave interference which creates the band gap of the PnC[7]. Two critical parameters determine the behavior and characteristics of the band gap[8]: the center frequency and band gap width. The center frequency is defined principally by the characteristic unit cell length $a$, typically on the order of the propagating wave's wavelength. The width of the band gap is heavily affected by the acoustic impedance discrepancy between the propagating medium and the inclusions. Filling factor (the ratio of the area of the inclusions to the area of the unit cell) is the primary parameter influencing the width of the gap. A structure's bandgap behavior can be described by its $f \cdot a$ product $[\mathrm{Hz} \cdot \mathrm{m}]$, as wavelength and frequency are inversely proportional. With increasing filling factor, the bandgap widens. Our design implements a honeycomb lattice, previously shown to offer the widest bandgap at the lowest filling factor (Fig.3), conferring exemplary decoupling while maintaining device robustness. FEM eigenmode simulations based on Bloch-Floquet wave theory were performed across the first Brillouin zone of the honeycomb lattice, with the eigenmodes subsequently mapped onto k-space yielding the bandgap[9].

The PnC design addresses the in-plane components of the energy lost to the substrate. However, without proper vertical outof-plane decoupling, anchor loss remains a significant issue. To enable vertical confinement, it is critical to match the thickness of the silicon handle layer to the quarter wavelength of the acoustic wave[10], determined by its frequency and the speed of wave propagation in silicon (Fig.4). By suspending the device in air to maximize acoustic impedance mismatch, the quarter wavelength roughly creates a standing wave with node and antinode at the surfaces of the handle layer, confining energy and elevating $Q_{\mathrm{ANC}}$.

In perfectly matched layer (PML) simulation, the tandem of quarter wavelength matching and the $\mathrm{PnC}$ offer excellent substrate decoupling. Critically, placing more PnC unit cells in the decoupling layer offers greater opportunity for propagating waves to reflect and interfere, improving energy confinement. With a sufficient number of unit cells placed around the anchor of the disk, the disk can be made substrate "deaf," leaving the device limited exclusively by intrinsic dissipation mechanisms i.e. TED and AKE (Fig.5).

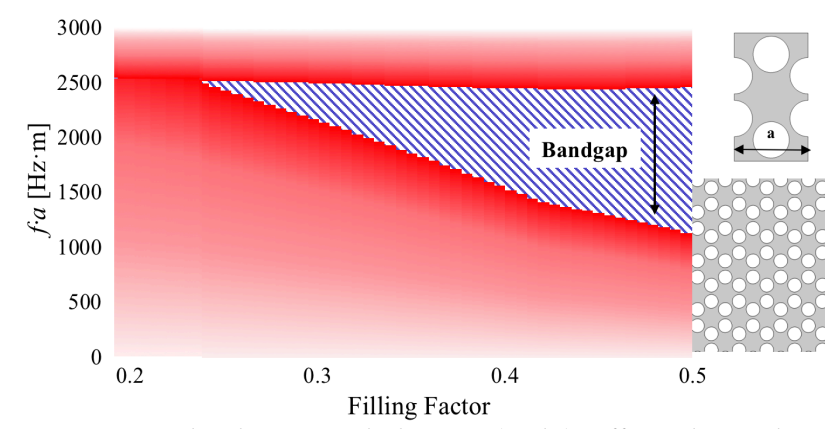

Figure 3: The honeycomb lattice (right) offers the widest bandgap at the smallest filling factor. The unit cell, when placed in a rectangular lattice, creates a honeycomb tessellation (bottom right).

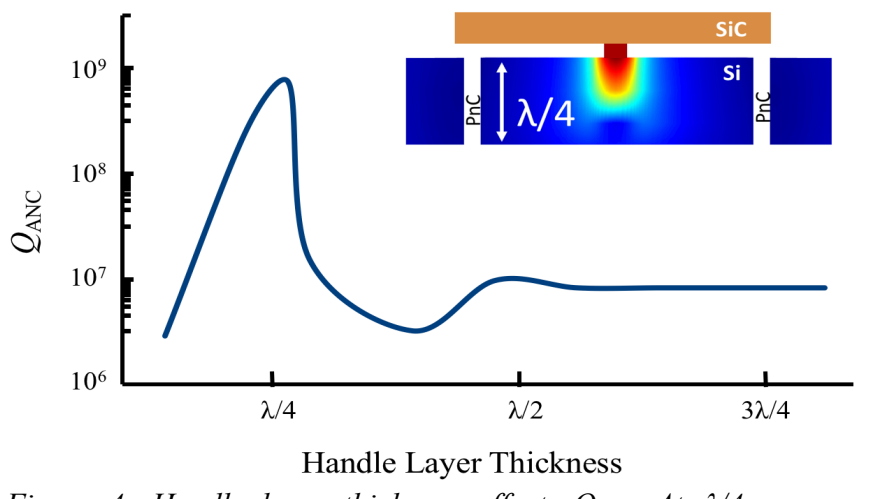

Figure 4: Handle layer thickness affects $Q_{A N C}$. At $\lambda / 4$, wave interference creates a standing wave with a node at the suspended end of the device. In concert with a PnC, anchor loss can be made negligible.

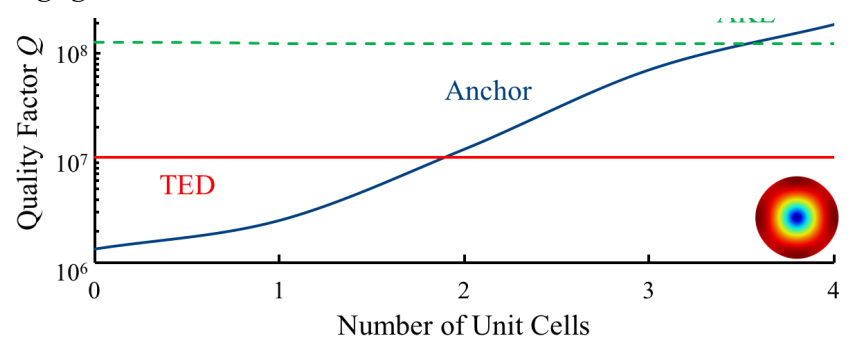

Figure 5: As more unit cells are added, $Q_{\text {Anchor }}($ blue) surpasses $Q_{T E D}$ (red) and finally $Q_{\text {Akhiezer }}$ (dashed green). In a $1540 \mu \mathrm{m}$ diameter disk, radial mode at $5 \mathrm{MHz}$ is TED limited.

\section{DEVICE DESIGN AND FABRICATION}

SiCOI substrates with $100 \mu \mathrm{m} \mathrm{SiC} \mathrm{device} \mathrm{layer} \mathrm{on} \mathrm{a} 500 \mu \mathrm{m} \mathrm{Si}$ handle layer separated by a thermally grown silicon oxide interlayer were custom-made in a process described in [11]. Complicating substrate choice, silicon carbide has an abundance of polytypes with cubic, hexagonal and rhombohedral symmetries. Among commercially available polytypes, atomically smooth monocrystalline 4H-SiC (hexagonally symmetric) wafers from Cree ${ }^{\circledR}$ exhibit the greatest isotropicity, making it better suited to demonstrate ultra-high $Q$ gyroscopic modes in center-supported disk resonators. Notably, the $\mathrm{m}=3$ bulk elliptical gyroscopic modes are fully degenerate in $4 \mathrm{H}-\mathrm{SiC}$, critical to mode-matched gyroscopic operation to take full advantage of $Q$.

After deposition of $\mathrm{Ti} / \mathrm{Cu}$ seed layer, the electroplated nickel mask is formed to define the disk resonators; the $\lambda / 4$ requirement prescribes the disk diameter to place the mode of interest at $5 \mathrm{MHz}$. Nickel is used as a hard mask for the subsequent SiC DRIE step, because of its high selectivity over $\mathrm{SiC}$, near 100:1, dwarfing that of $\mathrm{SiO}_{2}(\sim 1: 1)$. The disks are etched in an STS AOE chamber using a mixture of sulfur hexafluoride $\left(\mathrm{SF}_{6}\right)$ and Argon (Ar) with a large $\mathrm{DC}$ bias voltage. Carbon atoms in the $\mathrm{SiC}$ react with $\mathrm{SF}_{6}$, forming an inherent passivation layer $\left(\mathrm{C}_{\mathrm{x}} \mathrm{F}_{\mathrm{y}}\right)$, enabling anisotropic etching. Proper optimization allows for scallop-less trenches with superb anisotropy $(\sim 20: 1)$, albeit at greatly reduced etch rates ( $\sim 10 \mathrm{x}$ slower than in Si). Further, without cycling between etching and passivation steps, control of the trench profile requires fastidious optimization. The remaining nickel mask and any deposition on the sidewall are removed by wet etch. One concern rests in the fact that there is no etch stop, contrary to SOI processing where the BOX layer shows high selectivity over silicon. Conversely, silicon oxide shows no selectivity over silicon carbide, therefore proper timing is 
required for a consummate etch.

The PnC geometry is then defined via backside alignment to the $\mathrm{SiC}$ layer and etched by a standard Bosch process. This backside $500 \mu \mathrm{m}$ through-etch produces large amounts of Teflon which can exacerbate anchor losses if not properly cleaned. Using a material with higher acoustic velocity for the handle layer would relax this constraint. Finally, the sacrificial buried oxide layer is laterally etched with $\mathrm{HF}$ through the $\mathrm{PnC}$ structures doubling as release holes, self-aligning the pedestal with a size controlled by release time. The process yields a breathing mode disk resonator $(1540 \mu \mathrm{m})$ supported by a $\mathrm{SiO}_{2}$ pedestal $100 \mu \mathrm{m}$ ( $6 \%$ of disk size) in diameter (Fig. 6). It is anchored upon a phononic crystal composed of three unit cells, engineered with a unit cell length of $405 \mu \mathrm{m}$ to decouple the $5 \mathrm{MHz}$ radial mode. The pedestal is $2 \mu \mathrm{m}$ tall, separating the disk from the silicon handle layer below.

\section{MEASUREMENT AND RESULTS}

The fabricated disks are then mounted upon ultrasonic lithium niobate shear mode piezostages, adhered with Crystalbond. The setup is placed in a vacuum chamber and pumped down to submTorr. S21 frequency response measurements were taken with a laser Doppler vibrometer in conjunction with a network analyzer. Three devices of varying diameter were tested, each aiming to position the radial, $\mathrm{m}=3$, and $\mathrm{m}=4$ modes at $5 \mathrm{MHz}(1.56 \mathrm{~mm}$, $1.7 \mathrm{~mm}, 2.21 \mathrm{~mm}$ respectively). Notably, the $1.56 \mathrm{~mm}$ disk displayed a breathing mode $Q$ of $3.7 \mathrm{M}$ at $5 \mathrm{MHz}$ (Fig. 7); to date, this is the highest $f \cdot Q$ product $\left(1.85 \times 10^{13} \mathrm{~Hz}\right)$ measured in $\mathrm{SiC}$ resonators. Simulated with $Q_{\mathrm{ANC}}=350 \mathrm{M}$ and a limiting $Q_{\mathrm{TED}}=14 \mathrm{M}$ at $5 \mathrm{MHz}$, the $Q$ discrepancy arises due to sidewall defects (discussed later). In contrast, a similar disk (5mm diameter) anchored upon an unmodified silicon substrate (without $\mathrm{PnC}$ ) with smaller anchor diameter ( $3 \%$ of the device diameter) demonstrated a $Q$ of $\sim 1 \mathrm{M}$ at 1.7 MHz. Despite its larger size (less TED) and diminished pedestal area, the lack of any substrate decoupling bounds its $Q$ to smaller values.

Vibrational modes inside the bandgap show noticeably higher $Q$ 's relative to modes exterior to the gap (Fig. 8), though the radial mode outperforms the others. While the radial mode frequency was accurately simulated, other bulk elliptical mode frequencies systematically diverged from simulation, up to $6 \%$. Nevertheless, measured frequency splits $(\Delta f)$ are low for measured degenerate elliptical modes (Fig. 9), showing 4H-SiC's suitability for modematched gyroscopic operation.

\section{DISCUSSION}

The resonance modes, while substrate-decoupled, deviate slightly from TED simulation, which is the predicted limiting mechanism. Upon examination, the disk showed fabrication nonidealities such as horizontal and vertical striation. Previous work has shown defects and asperities in shell resonators significantly contribute to TED[12]. While shells with surface roughness show Qs below 100,000[13], attributable to a high surface area to volume ratio, the effect of surface defects on disks is less explored.

SEMs of the sidewall of the disks show striations on the micron scale. $Q_{\text {TED }}$ monotonically decreases with increasing size, flattening to $Q \mathrm{~s}$ in the millions. Horizontal striations appear when etching is disrupted and restarted, more easily obviated. Vertical striations however, could arise from a fusion of different sources: nonuniformities in the nickel mask that propagate during DRIE, in combination with charging in the passivation layer that segregates and directs plasma species imperfectly, result in a striated sidewall. In simulation, striations are modeled as a hemi-cylindrical defect on the sidewall of the disk, introducing additional strain coupling to thermal fields (Fig 10). Current fabricated SiC disk resonators show

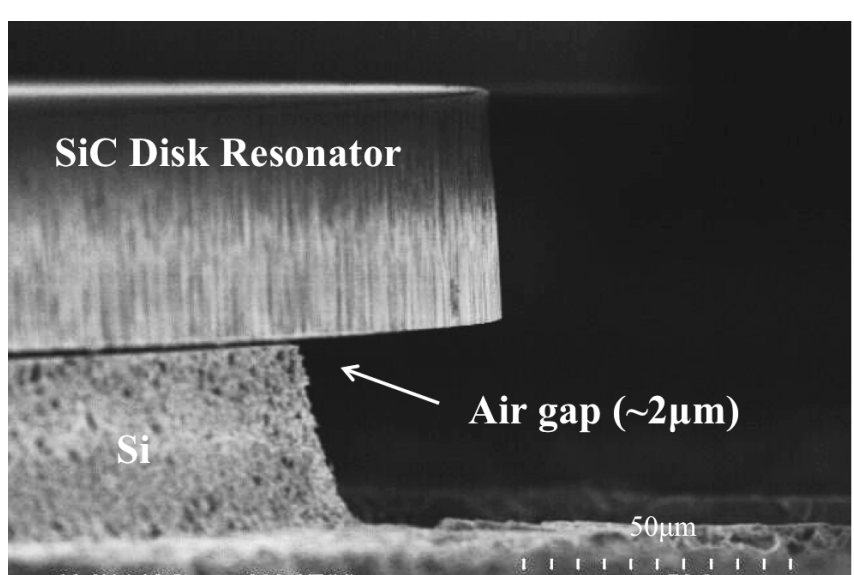

Figure 6: The device rests upon the silicon handle layer with $2 \mu \mathrm{m}$ gap determined by the thickness of the BOX layer. The lack of an etch stop manifests itself as over-etch into the silicon handle layer.

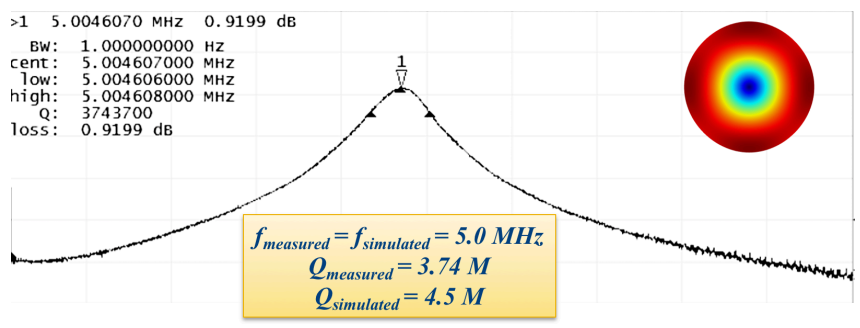

Figure 7: Radial mode frequency response of a 1540 $\mu$ m diameter disk with $f \cdot Q$ product of $1.85 \times 10^{13} \mathrm{~Hz}$. Measured $Q$ and f corroborate simulation well. $Q_{\text {simulated }}$ incorporates fabrication defects.

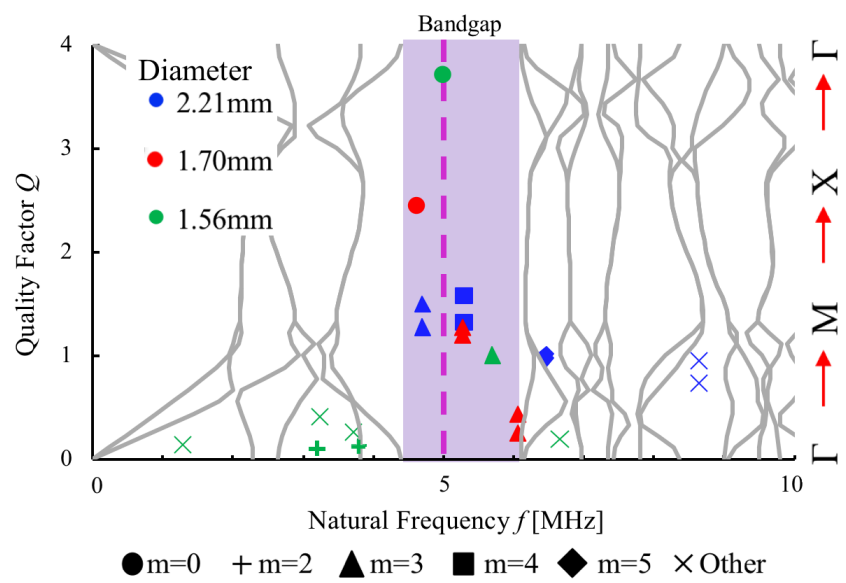

Figure 8: Simulated dispersion curves (gray) depict frequencies of allowed propagation across the first Brillouin zone. Measured $Q$ 's of bulk modes across devices of varying size inside and outside the bandgap (purple). The breathing mode $(m=0)$ exhibits $Q$ of $3.7 M$ (green circle), perfectly coinciding with $\lambda / 4$ frequency at $5 \mathrm{MHz}$ (dashed pink). Modes operating away from the $\lambda / 4$ frequency $(m=3$ modes at $5.3 \mathrm{MHz}$ ) suffer in performance.

defects ranging from $1-3 \mu \mathrm{m}$ in lateral dimension (Fig. 11). TED simulations incorporating these defects yield a $Q_{\text {TED }}$ of $4.5 \mathrm{M}$ for the radial mode at $5 \mathrm{MHz}$ (Fig. 7), confirming measurement as a TED limited mode.

Further discrepancies in $Q$-particularly for the $\mathrm{m}=3$ modescan be attributed to anchor losses due to fabrication and material non-idealities affecting the handle layer thickness, the disk frequency, and the PnC itself. Measured and simulated frequencies 


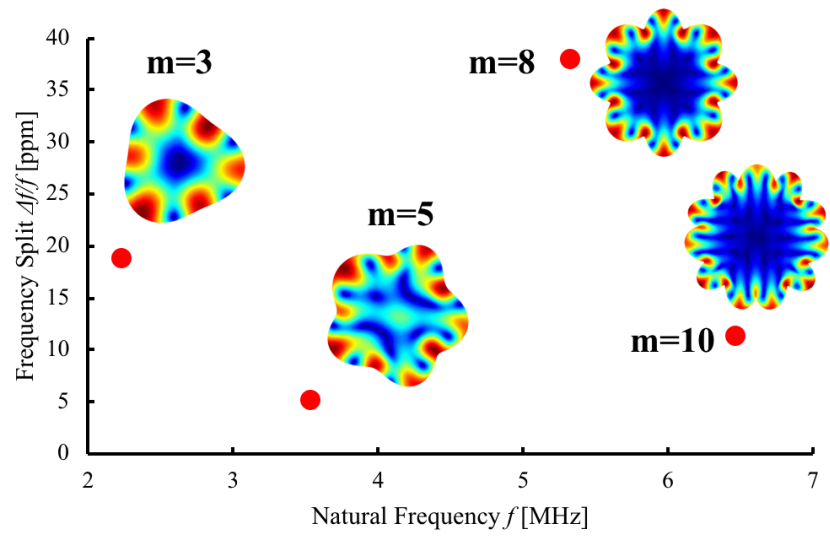

Figure 9: Measured frequency split $\Delta f / f$ of several bulk elliptical modes below 40 ppm. $M=3$ modes exhibit 18ppm frequency split.

differ by up to $6 \%$; e.g. the $\mathrm{m}=3$ degenerate modes were measured to be at $5.3 \mathrm{MHz}$ rather than $5 \mathrm{MHz}$ as simulated (Fig. 8). Due to strong $\lambda / 4$ dependence, operating at $5 \mathrm{MHz}(\mathrm{c}=\lambda \cdot f)$ is critical; in this case, resonance at $5.3 \mathrm{MHz}$ rather than $5 \mathrm{MHz}$ reduces $Q_{\mathrm{ANC}}$ by a factor of $3(100 \mathrm{M}->35 \mathrm{M})$. Additionally, the dimensions of the tethers between occlusions in the $\mathrm{PnC}$ should be tightly controlled to avoid spurious modes in the handle layer near $5 \mathrm{MHz}$. As opposed to other bulk elliptical modes, the radial mode showed the greatest consistency with simulation in terms of frequency and $Q$. Potential sources of this disagreement such as residual stresses during fabrication or material properties will be explored.

\section{CONCLUSION}

Centrally-supported BAW disk resonators with nano-scale sidewall roughness fabricated on SiCOI offer a pathway to extremely high $Q$ 's. Monocrystalline $\mathrm{SiC}$ BAW resonators offer low intrinsic material dissipation. Consequently, implementing a 3D substrate-decoupled handle layer-leaving the disk itself unblemished - unlocks the possibility of fabricating disk resonators with $Q$ 's tethered to intrinsic quantum limits in excess of $100 \mathrm{M}$.

In this work, surface TED limits the radial breathing mode to a $Q$ of $4 \mathrm{M}$ at $5 \mathrm{MHz}$. We are investigating several paths to improve $Q$ including $\mathrm{H}_{2} / \mathrm{Ar}$ annealing, roughness-optimized DRIE and SiCOI handle layer materials with lower sound velocity to allow more unit cells and thinner handle layer. Further, material and design anisotropy effects on frequency and $Q$ mismatches between gyroscopic modes will be scrutinized.

Harnessing these degenerate modes of $\mathrm{SiC}$ disk resonators for use in capacitively actuated gyroscopes presents its own unique challenges, along with remarkable rewards. This work represents a significant step forward in the pursuit of ultra-high $Q$ with a view toward achieving inertial grade BAW gyroscopes with superior vibration resilience in a small form factor.

\section{ACKNOWLEDGEMENT}

This work is supported by the Defense Advanced Research Projects Agency Microsystems Technology Office, Advanced Inertial Micro Sensors (AIMS) program and U.S. Navy (SPAWAR) under grant \#N66001-16-1-4027. The authors wish to thank the cleanroom staff at Georgia Tech IEN for their assistance.

\section{REFERENCES}

[1] F. Ayazi, "Multi-DOF inertial MEMS: From gaming to dead reckoning," in 2011 16th International Solid-State Sensors, Actuators and Microsystems Conference, 2011, pp. 2805-2808.

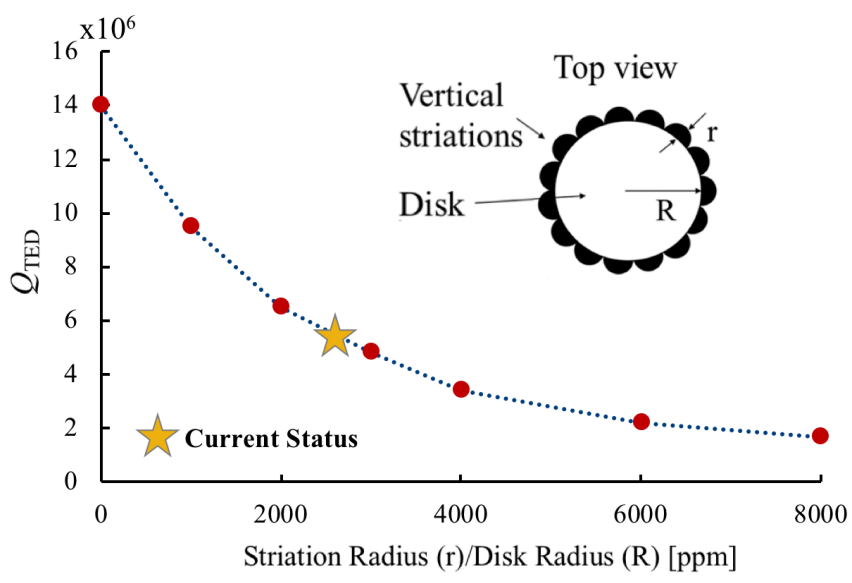

Figure 10: With increasing striation radius/disk radius ratio, simulation shows a precipitous reduction in $Q_{T E D}$ of the radial mode.

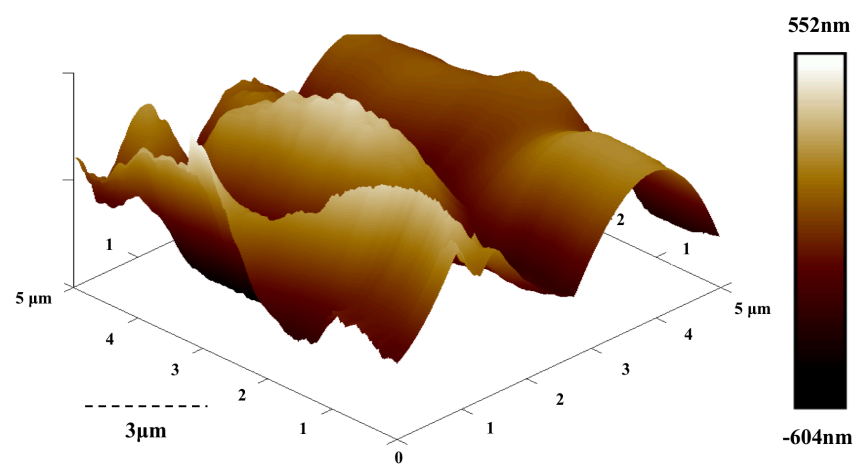

Figure 11: Atomic force micrograph of the sidewall of the disk showing striation and roughness on the micron scale.

[2] F. Ayazi, L. Sorenson, and R. Tabrizian, "Energy dissipation in micromechanical resonators," presented at the Micro- and Nanotechnology Sensors, Systems, and Applications III, 2011, vol. 8031, p. 803119.

[3] S. Ghaffari et al., "Quantum Limit of Quality Factor in Silicon Micro and Nano Mechanical Resonators," Sci. Rep., vol. 3, p. srep03244, Nov. 2013.

[4] R. Lifshitz and M. L. Roukes, "Thermoelastic damping in micro- and nanomechanical systems," Phys. Rev. B, vol. 61, no. 8, pp. 5600-5609, Feb. 2000 .

[5] D. E. Serrano et al., "Substrate-decoupled, bulk-acoustic wave gyroscopes: Design and evaluation of next-generation environmentally robust devices," Microsyst. Nanoeng., vol. 2, p. micronano201615, Apr. 2016.

[6] R. Mirjalili, H. Wen, D. E. Serrano, and F. Ayazi, "Substrate-decoupled silicon disk resonators having degenerate gyroscopic modes with $\mathrm{Q}$ in excess of 1 million," in 18th Conference on Solid-State Sensors, Actuators and Microsystems, 2015, pp. 15-18.

[7] M. Maldovan, "Sound and heat revolutions in phononics," Nature, vol. 503, no. 7475, pp. 209-217, Nov. 2013.

[8] A. Khelif and A. Adibi, Eds., Phononic Crystals. New York, NY: Springer New York, 2016.

[9] Y. Tsaturyan, et al., "Ultracoherent nanomechanical resonators via soft clamping and dissipation dilution," Nat. Nanotechnol., vol. 12, no. 8, p. nnano.2017.101, Jun. 2017.

[10] F. D. Bannon, J. R. Clark, and C. T. C. Nguyen, "High-Q HF microelectromechanical filters," IEEE J. Solid-State Circuits, vol. 35, no. 4, pp. 512-526, Apr. 2000

[11] S.-D. Ko, B. Hamelin, J. Yang, and F. Ayazi, "High-Q Monocrystalline Silicon Carbide Disk Resonators Fabricated Using DRIE of Thick SiC-On-Insulator Substrates," in The 31st IEEE International Conference on Micro Electro Mechanical Systems (MEMS 2018), 2018.

[12] L. Sorenson, P. Shao, and F. Ayazi, "Bulk and Surface Thermoelastic Dissipation in Micro-Hemispherical Shell Resonators," $J$. Microelectromechanical Syst., vol. 24, no. 2, pp. 486-502, 2015.

[13] B. Hamelin, V. Tavassoli, and F. Ayazi, "Microscale pierced shallow shell resonators: A test vehicle to study surface loss," in 2017 IEEE 30th International Conference on Micro Electro Mechanical Systems (MEMS), 2017, pp. 1134-1137. 